\title{
Super-energy tensor for space-times with vanishing scalar curvature
}

\author{
Miguel Á.G. Bonillat㘻 and Carlos F. Sopuerta ${ }^{\S}$ \\ ‡ Departament de Física Fonamental, Universitat de Barcelona, \\ Avda. Diagonal 647, E-08028 Barcelona, Spain \\ $\S$ Institut for Theoretical Physics, FSU Jena, \\ Max-Wien-Platz 1, D-07743 Jena, Germany
}

April 27, 2018

\begin{abstract}
A four-index tensor is constructed with terms both quadratic in the Riemann tensor and linear in its second derivatives, which has zero divergence for spacetimes with vanishing scalar curvature. This tensor reduces in vacuum to the Bel-Robinson tensor. Furthermore, the completely timelike component referred to any observer is positive, and zero if and only if the space-time is flat (excluding some unphysical space-times). We also show that this tensor is the unique that can be constructed with these properties. Such a tensor does not exist for general gravitational fields. Finally, we study this tensor in several examples: the Friedmann-Lemaitre-Robertson-Walker space-times filled with radiation, the plane-fronted gravitational waves, and the Vaidya radiating metric.
\end{abstract}

PACS Numbers: 04.20.-q, 04.20.Cv

\section{Introduction}

The investigation of conservation laws in general relativity has a long history. From its very beginning much of this research was based on pseudo-tensors instead of fully covariant methods. The aim of many of these works was to find differential laws which, once transformed into integral ones, were interpreted as energy balances in such a way that expressions for the energy and momentum densities of the gravitational field could be identified.

*Also at Laboratori de Física Matemàtica, Societat Catalana de Física, IEC, Barcelona. E-mail: mangel@ffn.ub.es, cfs@tpi.uni-jena.de 
The covariant approach to this problem is mainly based on the analogy of the Bel-Robinson tensor [1] with the energy-momentum tensor of the electromagnetic field (there are other approaches based on this analogy, see for instance [2]). The Bel-Robinson tensor is conserved in vacuum, completely symmetric and traceless. Moreover, the completely timelike component referred to any observer (described by a timelike unit vector field) is non-negative, and its vanishing implies that the spacetime is conformally flat (flat in vacuum). This is a desirable positivity property for any candidate to gravitational energy density. In spite of these good properties, the Bel-Robinson tensor has dimensions of energy density square and this fact makes its interpretation somewhat unclear. Nevertheless, it has revealed as a very useful tool in many kinds of studies, which has led to some efforts in finding extensions of the Bel-Robinson tensor for more general cases than vacuum. Therefore, the question arises whether generalizations of the Bel-Robinson tensor exist for space-times not necessarily empty.

The Bel tensor [3] was the first attempt on this problem. It is a tensor whose completely timelike component is positive and zero only when the space-time is Minkowski. In vacuum, it reduces to the Bel-Robinson tensor, but in the general case it is no longer conserved.

For general space-times, Sachs [4] found a divergence-free tensor that coincides with the Bel-Robinson tensor in vacuum. Unfortunately, this tensor does not satisfy any positivity property and it is neither completely symmetric nor traceless.

The systematic treatment of this problem was made by Collinson [5], who found all four-index divergence-free tensors with terms either quadratic in the Riemann tensor or linear in its second derivatives. The result found out is that any such tensor can be derived from only one tensor, namely $T_{10}^{\alpha \beta \lambda \mu}$ (see (17) in the appendix A), whose divergence with respect to the first index vanishes. However, there is not enough freedom to construct a tensor with its time component positive.

In this paper we show that, unlike the general case, for space-times with zero scalar curvature $(R=0)$ it is possible to construct a unique generalization of the Bel-Robinson tensor. We begin in Sect. 2 by proving that, when $R=0$, there exists another conserved tensor which cannot be derived from the Collinson one. In Sect. 3 we show that, demanding symmetry in the three free indices, there is not any other tensor independent from these two. This new tensor allows us to construct (Sect. 4) a divergence-free tensor which has the completely timelike component non-negative and zero only when the space-time is flat (excluding some cases that, via Einstein's field equations, have an unphysical matter content). This tensor is completely symmetric in its three last indices, but it is impossible to get a similar tensor symmetric in all their indices. We remark that it is not possible to construct any other tensor with such characteristics.

In order to illustrate this development, we study in Sect. 5 some examples in which we can define this tensor, namely: the Friedmann-Lemaitre-Robertson-Walker (FLRW) models with a energy-momentum content of (incoherent) radiation $(p=\varrho / 3)$, the plane-fronted gravitational waves with parallel rays ( $p p$ waves), and the Vaidya 
radiating space-time.

Finally, we recall that for purely electromagnetic space-times, and supposing that the Einstein field equations hold, Penrose and Rindler [6] also gave a generalization of the Bel-Robinson tensor by using spinor methods. This tensor is conserved, completely symmetric and traceless in its three last indices. In the appendix B we find its tensorial expression and a new (up to our knowledge) positivity property.

\section{Deduction of the new conserved tensor for $R=0$}

Otherwise said, throughout this paper we will consider the metric tensor $g_{\alpha \beta}$ to have signature $(-,+,+,+)$. The convention for indices on the Riemann tensor that will be used is defined through the Ricci identities:

$$
\left(\nabla_{\alpha} \nabla_{\beta}-\nabla_{\beta} \nabla_{\alpha}\right) v_{\lambda}=-R_{\lambda \alpha \beta}^{\sigma} v_{\sigma}
$$

where $v_{\alpha}$ is an arbitrary 1 -form. The Ricci tensor and the scalar curvature are defined as usual: $R_{\alpha \beta} \equiv R_{\alpha \sigma \beta}^{\sigma}$ and $R \equiv R_{\sigma}^{\sigma}$. We also recall the Riemann symmetries and the first and second Bianchi identities:

$$
\begin{gathered}
R_{\alpha \beta \lambda \mu}=R_{[\alpha \beta][\lambda \mu]}=R_{\lambda \mu \alpha \beta}, \\
R_{[\alpha \beta \lambda] \mu}=0 \\
\nabla_{[\nu} R_{\alpha \beta] \lambda \mu}=0 .
\end{gathered}
$$

The procedure we are going to use to find the conserved tensor starts from the expression for the divergence of the Bel tensor [1]:

$$
\nabla_{\alpha} T^{\alpha \beta \lambda \mu}=R_{\rho \sigma}^{\beta \lambda} J^{\mu \sigma \rho}+R_{\rho \sigma \sigma}^{\beta \mu} J^{\lambda \sigma \rho}-\frac{1}{2} g^{\lambda \mu} R_{\rho \sigma \gamma}^{\beta} J^{\sigma \gamma \rho}
$$

where the Bel tensor $T^{\alpha \beta \lambda \mu}$ and $J^{\alpha \beta \lambda}$ are defined as follows:

$$
\begin{aligned}
T^{\alpha \beta \lambda \mu} & \equiv \frac{1}{2}\left(R^{\alpha \rho \lambda \sigma} R_{\rho \sigma}^{\beta \mu}+* R *^{\alpha \rho \lambda \sigma} * R *_{\rho \sigma}^{\beta \mu}+* R^{\alpha \rho \lambda \sigma} * R_{\rho \sigma}^{\beta \mu}+R *^{\alpha \rho \lambda \sigma} R *_{\rho}^{\beta \mu}{ }_{\sigma}\right) \\
J^{\lambda \mu \beta} & \equiv \nabla^{\lambda} R^{\mu \beta}-\nabla^{\mu} R^{\lambda \beta}=\nabla_{\sigma} R^{\mu \lambda \beta \sigma}
\end{aligned}
$$

being “*” the usual dual operator acting over any pair of antisymmetric indices:

$$
\begin{gathered}
* R_{\alpha \beta \lambda \mu} \equiv \frac{1}{2} \eta_{\alpha \beta \sigma \rho} R_{\lambda \mu}^{\sigma \rho}, \quad R *_{\alpha \beta \lambda \mu} \equiv \frac{1}{2} \eta_{\lambda \mu \sigma \rho} R_{\alpha \beta}^{\sigma \rho} \\
* R *_{\alpha \beta \lambda \mu} \equiv \frac{1}{4} \eta_{\alpha \beta \gamma \delta} \eta_{\lambda \mu \sigma \rho} R^{\gamma \delta \sigma \rho}
\end{gathered}
$$

and $\eta_{\alpha \beta \lambda \mu}$ is the canonical volume 4 -form. Our purpose now is to work out the right hand side (r.h.s.) of equation (3) in order to convert it into a global divergence. To that end, we will repeatedly integrate by parts and make use of equations (1, 2). 
To begin with, the last term in equation (3) can be easily transformed into a divergence, by means of the Ricci and Bianchi identities (11,2):

$$
-\frac{1}{2} g^{\lambda \mu} R_{\rho \sigma \gamma}^{\beta} J^{\sigma \gamma \rho}=-g^{\lambda \mu} \nabla_{\alpha} \nabla_{\rho} J^{\alpha \rho \beta} .
$$

Next we expand the leading terms of the r.h.s. of (3) by using the definition of $J^{\alpha \beta \lambda}$ (1) and integrating by parts:

$$
R_{\rho \sigma}^{\beta \lambda} \nabla^{\mu} R^{\sigma \rho}-\nabla_{\alpha}\left(R^{\mu \rho} R_{\rho}^{\beta \lambda \alpha}\right)+R^{\mu \rho} \nabla_{\alpha} R_{\rho}^{\beta \lambda}+[\lambda \longleftrightarrow \mu]
$$

The first term in the previous expression can be rewritten by means of the Ricci identities (1) as follows:

$$
\begin{array}{r}
R_{\rho \sigma}^{\beta \lambda} \nabla^{\mu} R^{\sigma \rho}=\left(\nabla^{\lambda} \nabla_{\sigma}-\nabla_{\sigma} \nabla^{\lambda}\right) \nabla^{\mu} R^{\sigma \beta}+ \\
+\left[-\nabla^{\alpha}\left(R_{\alpha{ }_{\sigma}{ }^{\mu} R^{\sigma \beta}}\right)+R^{\sigma \beta} \nabla^{\alpha} R_{\sigma \alpha}^{\lambda \mu}{ }^{\mu}\right]+R_{\sigma}^{\lambda} \nabla^{\mu} R^{\sigma \beta} .
\end{array}
$$

Thus, we have converted the r.h.s. of equation (3) into a divergence plus the following terms:

$$
\begin{array}{r}
R^{\sigma \beta} \nabla^{\alpha} R_{\sigma \alpha}^{\lambda \mu}+R^{\sigma \lambda} \nabla^{\mu} R_{\sigma}^{\beta}+R^{\mu \rho} \nabla_{\alpha} R_{\rho}^{\beta}{ }^{\lambda \alpha}+[\lambda \longleftrightarrow \mu]= \\
=R^{\sigma \beta}\left(\nabla_{\sigma} R^{\lambda \mu}-\nabla^{\lambda} R_{\sigma}^{\mu}\right)+R^{\sigma \lambda} \nabla^{\mu} R_{\sigma}^{\beta}+R^{\mu \rho}\left(\nabla_{\rho} R^{\beta \lambda}-\nabla^{\beta} R_{\rho}^{\lambda}\right)+[\lambda \longleftrightarrow \mu] .
\end{array}
$$

Now, taking into account the contracted Bianchi identities $\left(\nabla_{\mu} R^{\mu \nu}=\frac{1}{2} \nabla^{\nu} R\right)$, these terms can be transformed into the following expression:

$$
\begin{gathered}
-\nabla^{\beta}\left(R_{\sigma}^{\lambda} R^{\sigma \mu}\right)+\nabla^{\mu}\left(R_{\sigma}^{\lambda} R^{\sigma \beta}\right)+\nabla^{\lambda}\left(R_{\sigma}^{\mu} R^{\sigma \beta}\right)-2 R^{\beta \sigma}\left(\nabla^{\mu} R_{\sigma}^{\lambda}+\nabla^{\lambda} R_{\sigma}^{\mu}\right)+ \\
+\nabla_{\sigma}\left(R^{\beta \lambda} R^{\mu \sigma}+R^{\beta \mu} R^{\lambda \rho}+2 R^{\beta \sigma} R^{\lambda \mu}\right)-\frac{1}{2}\left(R^{\beta \lambda} \nabla^{\mu} R+R^{\beta \mu} \nabla^{\lambda} R+2 R^{\lambda \mu} \nabla^{\beta} R\right) .
\end{gathered}
$$

The last three terms of this expression vanish when $R$ is constant, so we are finally left with $-2 R^{\beta \sigma}\left(\nabla^{\mu} R_{\sigma}^{\lambda}+\nabla^{\lambda} R_{\sigma}^{\mu}\right)$. Nevertheless, notice that our final purpose is to find a conserved tensor $T^{\prime \prime \alpha \beta \lambda \mu}$ whose completely timelike component referred to an observer $\vec{u}, T^{\prime \prime \alpha \beta \lambda \mu} u_{\alpha} u_{\beta} u_{\lambda} u_{\mu}$, is positive, which means that we are only interested in the symmetric part. Therefore, without lost of generality, we can symmetrize the whole expression and, as a consequence, the remaining terms transform themselves into a divergence:

$$
-4 R^{\sigma(\beta} \nabla^{\lambda} R_{\sigma}^{\mu)}=-2 \nabla^{(\beta}\left(R_{\sigma}^{\lambda} R^{\mu) \sigma}\right)
$$

So, we have finally achieved a conserved tensor if the scalar curvature vanishes (in fact, if it is constant). Collecting all the previous terms (5-10) we get the final result:

$$
\nabla_{\alpha} T^{\prime \prime \alpha \beta \lambda \mu}=-2 R^{(\beta \lambda} \nabla^{\mu)} R
$$


where we have defined

$$
\begin{aligned}
T^{\prime \prime \alpha \beta \mu} & \equiv T^{\alpha(\beta \lambda \mu)}-4 R^{\alpha(\beta} R^{\lambda \mu)}+g^{\alpha(\beta} R_{\sigma}^{\lambda} R^{\mu) \sigma}- \\
& -2 \nabla^{(\beta} \nabla^{\lambda} R^{\mu) \alpha}+2 \nabla^{(\beta} \nabla^{|\alpha|} R^{\lambda \mu)}-2 \nabla^{\alpha} \nabla^{(\beta} R^{\lambda \mu)}- \\
& -2 g^{\alpha(\beta} \nabla_{\sigma} \nabla^{\lambda} R^{\mu) \sigma}-\nabla_{\sigma} \nabla^{\sigma} R^{\alpha(\beta} g^{\lambda \mu)}+\nabla_{\sigma} \nabla^{\alpha} R^{\sigma(\beta} g^{\lambda \mu)}
\end{aligned}
$$

It is a matter of checking that this tensor cannot be obtained from Collinson tensor $T_{10}^{\alpha \beta \lambda \mu}$ when it is restricted to the case $R=0$. Now, we are left with the question of its uniqueness.

\section{Uniqueness}

In this section we will prove that, in the case we are concerned with $(R=0)$, it does not exist any other conserved tensor, symmetric in its three last indices, independent from $T^{\prime \prime \alpha \beta \lambda \mu}$ and the Collinson tensor, $T_{10}^{\alpha \beta \lambda \mu}$. The reasoning is the following. Suppose you are given a tensor $T^{\prime \prime \prime} \alpha \beta \lambda \mu$ which is conserved when $R=0$. The divergence computed in the general case will be a combination of the following type (taking into account symmetries and unit dimensions):

$$
\begin{gathered}
\nabla_{\alpha} T^{\prime \prime \prime \alpha \beta \lambda \mu}=a R^{(\beta \lambda} \nabla^{\mu)} R+b R \nabla^{(\beta} R^{\lambda \mu)}+c R^{\sigma(\beta} \nabla_{\sigma} R g^{\lambda \mu)}+d R g^{(\beta \lambda} \nabla^{\mu)} R+ \\
+e \nabla^{(\beta} \nabla^{\lambda} \nabla^{\mu)} R+f \nabla^{\sigma} \nabla_{\sigma} \nabla^{(\beta} R g^{\lambda \mu)}+h \nabla^{\sigma} \nabla^{(\beta} \nabla_{\sigma} R g^{\lambda \mu)}+i \nabla^{(\beta} \nabla^{|\sigma|} \nabla_{\sigma} R g^{\lambda \mu)}
\end{gathered}
$$

$a, b, c, d, e, f, h$ and $i$ being constants. This can be immediately cast in the following form:

$$
\nabla_{\alpha} T^{\prime \prime \prime \alpha \beta \lambda \mu}=(a-b) R^{(\beta \lambda} \nabla^{\mu)} R+\nabla_{\alpha} \tau^{\alpha \beta \lambda \mu}
$$

where $\tau^{\alpha \beta \lambda \mu}$ stands for:

$$
\begin{gathered}
\tau^{\alpha \beta \lambda \mu} \equiv b R g^{\alpha(\beta} R^{\lambda \mu)}+c\left(R R^{\alpha(\beta} g^{\lambda \mu)}-\frac{1}{4} g^{\alpha(\beta} g^{\lambda \mu)} R^{2}\right)+d \frac{1}{2} g^{(\beta \lambda} g^{\mu) \alpha} R^{2}+ \\
+e g^{\alpha(\beta} \nabla^{\lambda} \nabla^{\mu)} R+f g^{\alpha \sigma} \nabla_{\sigma} \nabla^{(\beta} R g^{\lambda \mu)}+h g^{\alpha \sigma} \nabla^{(\beta} \nabla_{\sigma} R g^{\lambda \mu)}+i g^{\alpha(\beta} \nabla^{|\sigma|} \nabla_{\sigma} R g^{\lambda \mu)}
\end{gathered}
$$

so clearly it is a tensor that vanishes when $R$ does. That is, if a tensor of the kind we are considering is divergence-free when $R=0$, in the general case its divergence should be a multiple of $R^{(\beta \lambda} \nabla^{\mu)} R$ plus the divergence of a tensor of the type $\tau^{\alpha \beta \lambda \mu}$. If we had two such tensors, a suitable combination of them removing the term $R^{(\beta \lambda} \nabla^{\mu)} R$ would give a conserved tensor for the general case and, therefore, due to the Collinson result [5], it could be constructed from $T_{10}^{\alpha \beta \lambda \mu}$. Given that the tensor $\tau^{\alpha \beta \lambda \mu}$ vanishes when $R=0$, the three tensors would not be independent in that case.

On the other hand, this reasoning shows that, from the very beginning, we were able to know that in the $R=0$ case at most one more conserved tensor could exist apart from Collinson's one, as finally has been the case. 


\section{Positivity}

As it has been pointed out above, in the general case all the conserved tensors can be constructed from $T_{10}^{\alpha \beta \lambda \mu}$. This construction is based in two procedures. First, it is clear that if we perform any permutation on the three last indices we will still have a conserved tensor. Second, by taking the two traces $T_{10}^{\alpha \beta \rho}, T_{10}^{\alpha \rho \beta}{ }_{\rho}$ and multiplying them by $g^{\lambda \mu}$ we obtain new conserved tensors. Actually, there is not any other two-index divergence-free tensor independent from them. These two tensors can be taken to be (as usually obtained by hamiltonian differentiation):

$$
\begin{aligned}
& t_{1}^{\alpha \beta}=2 \nabla^{\alpha} \nabla^{\beta} R-2 g^{\alpha \beta} \nabla_{\mu} \nabla^{\mu} R+\frac{1}{2} g^{\alpha \beta} R^{2}-2 R R^{\alpha \beta}, \\
& t_{2}^{\alpha \beta}=2 \nabla_{\sigma} \nabla^{\alpha} R^{\sigma \beta}-\nabla_{\sigma} \nabla^{\sigma} R^{\alpha \beta}-2 R^{\alpha \sigma} R_{\sigma}^{\beta}+\frac{1}{2} R_{\sigma \rho} R^{\sigma \rho} g^{\alpha \beta}-\frac{1}{2} g^{\alpha \beta} \nabla_{\mu} \nabla^{\mu} R .
\end{aligned}
$$

This is all the freedom we have in the general case.

In the $R=0$ case, there exists only one two-index conserved tensor, which is that obtained from $t_{2}^{\alpha \beta}$. On the other hand, as we consider tensors which are symmetric in the three last indices, from the Collinson tensor we will only have one independent tensor (apart from the two-index tensor), namely $T_{10}^{\alpha(\beta \lambda \mu)}$ or, equivalently, the Sachs tensor $T^{\prime \alpha \beta \lambda \mu}$ [4] which is symmetric in its three last indices.

Therefore, to construct the super-energy tensor in the $R=0$ case, we are led with three tensors: $i$ ) the Sachs tensor $T^{\prime \alpha \beta \lambda \mu}$ (restricted to the $R=0$ case):

$$
\begin{aligned}
T^{\alpha \beta \lambda \mu} & \equiv T^{\alpha(\beta \lambda \mu)}+2 R^{\alpha \sigma} R_{\sigma}^{(\beta} g^{\lambda \mu)}-\frac{1}{3} g^{\alpha(\beta} R_{\sigma}^{\lambda} R^{\mu) \sigma}-\frac{1}{2} R_{\sigma \rho} R^{\sigma \rho} g^{\alpha(\beta} g^{\lambda \mu)}+ \\
& +2 \nabla^{(\beta} \nabla^{\lambda} R^{\mu) \alpha}-\frac{2}{3} \nabla^{(\beta} \nabla^{|\alpha|} R^{\lambda \mu)}-\frac{2}{3} \nabla^{\alpha} \nabla^{(\beta} R^{\lambda \mu)}+ \\
& +\frac{4}{3} \nabla_{\sigma} \nabla^{\sigma} R^{(\beta \lambda} g^{\mu) \alpha}-2 g^{\alpha(\beta} \nabla_{\sigma} \nabla^{\lambda} R^{\mu) \sigma}-\nabla_{\sigma} \nabla^{\alpha} R^{\sigma(\beta} g^{\lambda \mu)},
\end{aligned}
$$

ii) the two-index tensor $t^{\alpha \beta}$ :

$$
t^{\alpha \beta}=2 \nabla_{\sigma} \nabla^{\alpha} R^{\sigma \beta}-\nabla_{\sigma} \nabla^{\sigma} R^{\alpha \beta}-2 R^{\alpha \sigma} R_{\sigma}^{\beta}+\frac{1}{2} R_{\sigma \rho} R^{\sigma \rho} g^{\alpha \beta}
$$

and iii) the tensor $T^{\prime \prime \alpha \beta \lambda \mu}$ previously found in (11).

Now, we have to combine these three tensors in such a way that any observer measures a positive quantity. Moreover, we would like that this completely timelike component vanishes if and only if the space-time is flat.

First of all, we have to take into account that terms made of derivatives of the Ricci tensor do not have a definite sign, so it would be necessary to eliminate their contributions. This aim can only be achieved by means of the following combination:

$$
A^{\alpha \beta \lambda \mu} \equiv \frac{1}{2}\left(3 T^{\prime \alpha \beta \lambda \mu}-T^{\prime \prime \alpha \beta \lambda \mu}\right)+\frac{5}{2} t^{\alpha(\beta} g^{\lambda \mu)},
$$


which more explicitly reads:

$$
\begin{aligned}
A^{\alpha \beta \lambda \mu} & =T^{\alpha(\beta \lambda \mu)}+ \\
& +2 R^{\alpha(\beta} R^{\lambda \mu)}-2 R^{\alpha \sigma} R_{\sigma}^{(\beta} g^{\lambda \mu)}-g^{\alpha(\beta} R_{\sigma}^{\lambda} R^{\mu) \sigma}+\frac{1}{2} R_{\sigma \rho} R^{\sigma \rho} g^{\alpha(\beta} g^{\lambda \mu)}+ \\
& +2 \nabla^{(\beta} \nabla^{\lambda} R^{\mu) \alpha}-2 \nabla^{(\beta} \nabla^{|\alpha|} R^{\lambda \mu)}+3 \nabla_{\sigma} \nabla^{\alpha} R^{\sigma(\beta} g^{\lambda \mu)}- \\
& -2 g^{\alpha(\beta} \nabla_{\sigma} \nabla^{\lambda} R^{\mu) \sigma}+2 \nabla_{\sigma} \nabla^{\sigma} R^{(\beta \lambda} g^{\mu) \alpha}-2 \nabla_{\sigma} \nabla^{\sigma} R^{\alpha(\beta} g^{\lambda \mu)} .
\end{aligned}
$$

Since we have already exhausted all the freedom, we finally examine the completely timelike component referred to any timelike unit vector $\vec{u}$ :

$$
\begin{aligned}
A(\vec{u}) & \equiv A_{\alpha \beta \lambda \mu} u^{\alpha} u^{\beta} u^{\lambda} u^{\mu}=T_{\alpha \beta \lambda \mu} u^{\alpha} u^{\beta} u^{\lambda} u^{\mu}+\frac{1}{2} R_{\sigma \rho} R^{\sigma \rho}+ \\
& +2\left(R_{\alpha \beta} u^{\alpha} u^{\beta}\right)^{2}+3\left(R^{\alpha \sigma} R_{\sigma}^{\beta}\right) u_{\alpha} u_{\beta}-\left(\nabla_{\sigma} \nabla^{\alpha} R^{\sigma \beta}\right) u_{\alpha} u_{\beta} .
\end{aligned}
$$

To check the positivity of $A(\vec{u})$ it is convenient to write out the last term of the previous expression in the following form:

$$
\nabla_{\sigma} \nabla^{\alpha} R^{\sigma \beta}=C_{\sigma \rho}^{\alpha \beta} R^{\sigma \rho}+2 R^{\alpha \sigma} R_{\sigma}^{\beta}-\frac{1}{2} g^{\alpha \beta} R_{\sigma \rho} R^{\sigma \rho} .
$$

At this point, we introduce four spatial tensors, namely $E_{\alpha \beta}(\vec{u}), H_{\alpha \beta}(\vec{u}), M_{\alpha \beta}(\vec{u})$ and $N_{\alpha \beta}(\vec{u})$, that (together with $R$ ) wholly characterize the Riemann tensor [7]. Their definitions, properties and some useful formulae are given in the appendix A.

Introducing the previous definitions in (13) we obtain, after some calculations:

$$
A(\vec{u})=\left(E_{\sigma \rho}-M_{\sigma \rho}\right)\left(E^{\sigma \rho}-M^{\sigma \rho}\right)+H_{\sigma \rho} H^{\sigma \rho}+3 N_{\sigma \rho} N^{\sigma \rho}+\left(R_{\alpha \beta} u^{\alpha} u^{\beta}\right)^{2},
$$

which is a sum of square terms (all the tensors appearing here are spatial). Therefore it is manifestly positive and its vanishing implies:

$$
\begin{array}{r}
H_{\alpha \beta}=0, \quad N_{\alpha \beta}=0, \\
R_{\alpha \beta} u^{\alpha} u^{\beta}=0, \quad E_{\alpha \beta}=M_{\alpha \beta} .
\end{array}
$$

The previous expressions lead to $R_{\alpha \beta} u^{\alpha}=0$ (see appendix A). This condition, when considering the Einstein field equations, immediately drives to an unphysical energymomentum tensor. Hence, if we eliminate these unphysical space-times (for instance, adding any energy condition), the vanishing of $A(\vec{u})$ finally implies the Minkowski space-time.

\section{Some examples}

In this section we are going to study the tensor $A^{\alpha \beta \lambda \mu}$ (12) in some space-times with vanishing scalar curvature. In particular, we are going to consider the following examples: (i) the radiation FLRW cosmological models, (ii) the $p p$ waves, and (iii) the 
Vaidya radiating metric. In examples (i) and (ii) we will give the expression for $A^{\alpha \beta \lambda \mu}$ and its completely timelike component $A(\vec{u})$ (14) for an arbitrary observer $\vec{u}$. In the example (iii), for the sake of brevity we will give only the expression of $A(\vec{u})$, also for an arbitrary observer.

In the first example we study the case of the FLRW models (see for instance [8]) with vanishing scalar curvature, the radiation models, whose energy-momentum tensor (of perfect-fluid type) is given by the following expression (throughout this section we will use units in which $8 \pi G=c=1$ )

$$
T_{\alpha \beta}=\varrho U_{\alpha} U_{\beta}+p h_{\alpha \beta}, \quad p=\frac{1}{3} \varrho,
$$

where $\vec{U}$ is the fluid velocity $\left(U^{\alpha} U_{\alpha}=-1\right), \varrho$ the energy density, $p$ the pressure, and $h_{\alpha \beta}=g_{\alpha \beta}+U_{\alpha} U_{\beta}$ the orthogonal projector to the fluid velocity. The line element of these conformally-flat models can be written as

$$
d s^{2}=-d t^{2}+a^{2}(t)\left\{d \chi^{2}+\Sigma^{2}(\epsilon, \chi)\left(d \theta^{2}+\sin ^{2} \theta d \varphi^{2}\right)\right\}
$$

where $\Sigma(\epsilon, \chi)$ is given by

$$
\Sigma(\epsilon, \chi)= \begin{cases}\sin \chi & \text { if } \epsilon=1 \\ \chi & \text { if } \epsilon=0 \\ \sinh \chi & \text { if } \epsilon=-1\end{cases}
$$

The fluid velocity $\vec{U}$, the scale factor $a(t)$ and the energy density $\varrho(t)$ are

$$
\vec{U}=\frac{\partial}{\partial t}, \quad a^{2}(t)=\left(t-t_{o}\right)\left[2 A-\epsilon\left(t-t_{o}\right)\right], \quad \varrho(t)=\frac{3 A^{2}}{a^{4}(t)},
$$

respectively, and $A$ and $t_{o}$ are arbitrary constants.

After some straightforward calculations, and using the special properties of these space-times, we arrive at the following expression for $A^{\alpha \beta \lambda \mu}$

$$
A^{\alpha \beta \lambda \mu}=\frac{4}{3} \varrho^{2}\left\{U^{\alpha} U^{\beta} U^{\lambda} U^{\mu}+U^{\alpha} U^{(\beta} h^{\lambda \mu)}+\frac{5}{3} h^{\alpha(\beta} U^{\lambda} U^{\mu)}+\frac{1}{3} h^{\alpha(\beta} h^{\lambda \mu)}\right\} .
$$

As we can see, it is proportional to the energy density squared. We can also check that it is indeed divergence-free. Now, let us compute the completely timelike component (14) of this tensor with respect to an arbitrary observer $\vec{u}$. To that end, we decompose $\vec{u}$ in the next way

$$
\vec{u}=\gamma(\vec{U}+\vec{v}), \quad v^{\alpha} U_{\alpha}=0, \quad v^{\alpha} v_{\alpha}=v^{2} \geq 0, \quad \gamma \equiv\left(1-v^{2}\right)^{-1 / 2},
$$

where the case $\vec{v}=0$ corresponds to an observer comoving with the fluid $(\vec{u}=\vec{U})$. Then, from (14, 15) we find that $A(\vec{u})$ is given by

$$
A(\vec{u})=\frac{4}{3} \varrho^{2} \gamma^{4}\left\{1+\frac{8}{3} v^{2}+\frac{1}{3} v^{4}\right\} .
$$


That is, it is function of $\varrho$ and $v$ only. Moreover, it increases monotonically as $v$ increases and its minimum corresponds to the case $v=0$, in which the observer is comoving with the fluid.

Now, we are going to study the tensor $A^{\alpha \beta \lambda \mu}$ in the case of the $p p$ waves spacetimes. The corresponding line element can be written in null coordinates $\{u, v, \zeta, \bar{\zeta}\}$ as follows (see [8] for details)

$$
d s^{2}=-2 d u d v+2 d \zeta d \bar{\zeta}-2 H d u^{2}
$$

where $H$ is an arbitrary function which does not depend on $v[H=H(u, \zeta, \bar{\zeta})]$. Using the following Newman-Penrose basis $\{\ell, k, m, \bar{m}\}$

$$
\vec{\ell}=\frac{\partial}{\partial v}, \quad \vec{k}=\frac{\partial}{\partial u}-H \frac{\partial}{\partial v}, \quad \vec{m}=\frac{\partial}{\partial \zeta},
$$

the Ricci and self-dual Weyl tensors are

$$
R_{\alpha \beta}=2 \Phi \ell_{\alpha} \ell_{\beta}, \quad \hat{C}_{\alpha \beta \lambda \mu} \equiv C_{\alpha \beta \lambda \mu}+i \stackrel{*}{C}_{\alpha \beta \lambda \mu}=2 \Psi_{4} V_{\alpha \beta} V_{\lambda \mu},
$$

respectively. Where the quantities $\Phi, \Psi_{4}$ and $V_{\alpha \beta}$ are given by

$$
\Phi \equiv H_{, \zeta \bar{\zeta}}, \quad \Psi_{4}=H_{, \bar{\zeta} \bar{\zeta}}, \quad V_{\alpha \beta} \equiv 2 \ell_{[\alpha} m_{\beta]}
$$

From (16) we can see that the energy-momentum content can correspond with vacuum, Einstein-Maxwell or pure radiation fields. Moreover, the Petrov type is N, being $\vec{\ell}$ the repeated principal direction of the Weyl tensor, which in fact is a constant vector field $\left(\nabla_{\alpha} \ell_{\beta}=0\right)$.

After some calculations, we have found that the Bel tensor $T^{\alpha \beta \lambda \mu}$ and our tensor $A^{\alpha \beta \lambda \mu}$ are

$$
\begin{gathered}
T^{\alpha \beta \lambda \mu}=4\left(\Phi^{2}+\Psi_{4} \bar{\Psi}_{4}\right) \ell^{\alpha} \ell^{\beta} \ell^{\lambda} \ell^{\mu} \\
\frac{1}{4} A^{\alpha \beta \lambda \mu}=\left(3 \Phi^{2}+\Psi_{4} \bar{\Psi}_{4}\right) \ell^{\alpha} \ell^{\beta} \ell^{\lambda} \ell^{\mu}+\ell^{\alpha} \ell^{(\beta} \nabla^{\lambda} \nabla^{\mu)} \Phi-\ell^{(\beta} \ell^{\lambda} \nabla^{\mu)} \nabla^{\alpha} \Phi \\
+\left[g^{\alpha(\beta} \ell^{\lambda} \ell^{\mu)}-\ell^{\alpha} \ell^{(\beta} g^{\lambda \mu)}\right] \nabla^{\sigma} \nabla_{\sigma} \Phi .
\end{gathered}
$$

From this expression, the completely timelike component is given by

$$
A(\vec{u})=4\left(3 \Phi^{2}+\Psi_{4} \bar{\Psi}_{4}\right)\left(\ell^{\alpha} u_{\alpha}\right)^{4} .
$$

Then, the vanishing of $A(\vec{u})$ implies the Minkowski space-time.

Finally, we are going to consider the Vaidya radiating space-time (see for instance [8]). For the sake of brevity we only give here the completely timelike component (14) of the tensor $A^{\alpha \beta \lambda \mu}$. The line element of this spherically symmetric metric can be written as follows

$$
d s^{2}=-2 F^{2}(u, v) d u d v+r^{2}(u, v)\left(d \theta^{2}+\sin ^{2} \theta d \varphi^{2}\right),
$$


where

$$
F^{2}(u, v)=f(u) \frac{\partial r}{\partial v}, \quad \frac{\partial r}{\partial u}=\frac{1}{2} f(u)\left(\frac{2 m(u)}{r}-1\right) .
$$

Here, $m(u)$ is the invariantly defined mass function. As is well-known, the Petrov type of this metric is D. Then, taking the following Newman-Penrose adapted basis

$$
\vec{\ell}=\frac{-1}{F} \frac{\partial}{\partial v}, \quad \vec{k}=\frac{-1}{F} \frac{\partial}{\partial u}, \quad \vec{m}=\frac{1}{\sqrt{2} r}\left(\frac{\partial}{\partial \theta}+\frac{i}{\sin \theta} \frac{\partial}{\partial \varphi}\right),
$$

where $\vec{\ell}$ and $\vec{k}$ are aligned with the principal directions of the Weyl tensor, the Ricci tensor is given by

$$
R_{\alpha \beta}=2 \Phi \ell_{\alpha} \ell_{\beta}, \quad \Phi \equiv-\frac{m_{, u}}{r^{2} r_{, u}} .
$$

And the only non-zero component of the Weyl tensor in this basis (see [《]) is

$$
\Psi_{2}=-\frac{m(u)}{r^{3}(u, v)},
$$

which in this case is real.

In terms of these quantities, we have found the following expression for $A(\vec{u})$

$$
\begin{gathered}
A(\vec{u})=2\left[\Psi_{2}+\Phi\left(\ell^{\alpha} u_{\alpha}\right)^{2}\right]^{2}+ \\
+4 \Psi_{2}^{2}\left[36\left(\ell^{\alpha} u_{\alpha}\right)^{2}\left(k^{\beta} u_{\beta}\right)^{2}-18\left(\ell^{\alpha} u_{\alpha}\right)\left(k^{\beta} u_{\beta}\right)+1\right]+10 \Phi^{2}\left(\ell^{\alpha} u_{\alpha}\right)^{4},
\end{gathered}
$$

and again, taking into account that

$$
2\left(\ell^{\alpha} u_{\alpha}\right)\left(k^{\beta} u_{\beta}\right) \geq 1 \quad \Longrightarrow \quad 36\left(\ell^{\alpha} u_{\alpha}\right)^{2}\left(k^{\beta} u_{\beta}\right)^{2}-18\left(\ell^{\alpha} u_{\alpha}\right)\left(k^{\beta} u_{\beta}\right)+1 \geq 1,
$$

$A(\vec{u})$ vanishes if and only if the space-time is the Minkowski space-time. When we restrict ourselves to observers lying on the 2-planes generated by the principal directions $\left[2\left(\ell^{\alpha} u_{\alpha}\right)\left(k^{\beta} u_{\beta}\right)=1\right]$, which are precisely the observers that minimize $A(\vec{u})$, the result is

$$
A(\vec{u})=2\left[\Psi_{2}+\Phi\left(\ell^{\alpha} u_{\alpha}\right)^{2}\right]^{2}+4 \Psi_{2}^{2}+10 \Phi^{2}\left(\ell^{\alpha} u_{\alpha}\right)^{4} .
$$

\section{Acknowledgments}

M.Á.G.B. gratefully acknowledges the Comissionat per a Universitats i Recerca de la Generalitat de Catalunya for financial support. C.F.S. gratefully acknowledges financial support in the form of a fellowship from the Alexander von Humboldt Foundation. 


\section{A Useful definitions}

We first write down the Collinson tensor [5]:

$$
T_{10}^{\alpha \beta \lambda \mu}=6 Q_{1}^{\alpha \beta \lambda \mu}+Q_{2}^{\alpha \beta(\lambda \mu)}+Q_{3}^{\alpha \beta(\lambda \mu)},
$$

where

$$
\begin{aligned}
Q_{1}^{\alpha \beta \lambda \mu}= & R_{\sigma}^{\lambda} R^{\beta \mu \alpha \sigma}+R_{\sigma}^{\beta} R^{\mu \alpha \lambda \sigma}+\nabla^{\mu} \nabla_{\sigma} R^{\alpha \sigma \beta \lambda}-\nabla^{\beta} \nabla_{\sigma} R^{\alpha \lambda \mu \sigma}-g^{\alpha \beta} \nabla_{\rho} \nabla_{\sigma} R^{\mu \sigma \lambda \rho}, \\
Q_{2}^{\alpha \beta \lambda \mu}= & -4 \nabla^{\lambda} \nabla_{\sigma} R^{\alpha \sigma \beta \mu}-6 \nabla^{\mu} \nabla_{\sigma} R^{\alpha \beta \lambda \sigma}-6 g^{\alpha \mu} \nabla_{\sigma} \nabla^{\lambda} R^{\sigma \beta}+ \\
& +6 g^{\alpha \mu} \nabla_{\rho} \nabla_{\sigma} R^{\sigma \lambda \beta \rho}+6 \nabla^{\mu} \nabla^{\lambda} R^{\alpha \beta}, \\
Q_{3}^{\alpha \beta \lambda \mu}= & -8 g^{\alpha \lambda} R_{\sigma \rho}{ }^{\mu}{ }_{\gamma} R^{\rho \gamma \beta \sigma}+8 R_{\sigma}{ }^{\lambda \mu}{ }_{\rho}^{\beta \sigma \alpha \rho}+8 R_{\sigma}^{\mu} R^{\beta \sigma \lambda \alpha}+8 R_{\sigma}{ }^{\beta \mu}{ }_{\rho} R^{\lambda \sigma \alpha}+ \\
& +2 R_{\sigma}^{\mu} R^{\beta \alpha \sigma \lambda}+2 R_{\sigma}{ }^{\mu \beta}{ }_{\rho} R^{\lambda \sigma \alpha \rho}+3 g^{\alpha \beta} R_{\sigma}^{\mu} R^{\lambda \sigma}-g^{\alpha \beta} R_{\sigma \rho}{ }^{\mu}{ }_{\gamma} R^{\rho \gamma \lambda \sigma},
\end{aligned}
$$

which is divergence-free in the index $\alpha$ and whose only symmetry on the indices $\beta, \lambda$ and $\mu$ is $T_{10}^{\alpha \beta[\lambda \mu]}-T_{10}^{\alpha \mu[\beta \lambda]}=0$.

Next, in order to introduce other useful definitions, recall the well-known decomposition of the Riemann tensor into its irreducible parts under the full Lorentz group:

$$
R_{\alpha \beta \lambda \mu}=C_{\alpha \beta \lambda \mu}+E_{\alpha \beta \lambda \mu}+G_{\alpha \beta \lambda \mu}
$$

where $C_{\alpha \beta \lambda \mu}$ is the Weyl tensor and

$$
\begin{aligned}
E_{\alpha \beta \lambda \mu} & \equiv \frac{1}{2}\left(\tilde{R}_{\alpha \lambda} g_{\beta \mu}-\tilde{R}_{\alpha \mu} g_{\beta \lambda}+\tilde{R}_{\beta \mu} g_{\alpha \lambda}-\tilde{R}_{\beta \lambda} g_{\alpha \mu}\right), \quad \tilde{R}_{\alpha \beta} \equiv R_{\alpha \beta}-\frac{1}{4} R g_{\alpha \beta}, \\
G_{\alpha \beta \lambda \mu} & \equiv \frac{R}{12}\left(g_{\alpha \lambda} g_{\beta \mu}-g_{\alpha \mu} g_{\beta \lambda}\right),
\end{aligned}
$$

being $R \equiv R_{\mu}^{\mu}$ the scalar curvature.

The electric and magnetic parts of the Weyl tensor associated to a timelike vector field $\vec{u}$ are:

$$
E_{\alpha \lambda}(\vec{u}) \equiv C_{\alpha \beta \lambda \mu} u^{\beta} u^{\mu}, \quad H_{\alpha \lambda}(\vec{u}) \equiv-\stackrel{*}{C}_{\alpha \beta \lambda \mu} u^{\beta} u^{\mu}
$$

These tensors are spatial (orthogonal to $\vec{u}$ ), symmetric and traceless, and they fully determine the Weyl tensor.

We can proceed analogously with the tensor $E_{\alpha \beta \lambda \mu}$ and define (see [7] for a more detailed study of these matters):

$$
M_{\alpha \lambda}(\vec{u}) \equiv E_{\alpha \beta \lambda \mu} u^{\beta} u^{\mu}, \quad N_{\alpha \lambda}(\vec{u}) \equiv-* E_{\alpha \beta \lambda \mu} u^{\beta} u^{\mu}
$$

We give here some of their properties, since they are less known than the electric and magnetic parts of the Weyl tensor:

$$
\begin{aligned}
M_{\alpha \lambda}= & M_{\lambda \alpha}, \quad M_{\alpha \lambda} u^{\lambda}=0, \quad M_{\mu}^{\mu}=\tilde{R}_{\mu \nu} u^{\mu} u^{\nu}, \\
& N_{\alpha \lambda}=-N_{\lambda \alpha}, \quad N_{\alpha \lambda} u^{\lambda}=0, \quad N_{\mu}^{\mu}=0 .
\end{aligned}
$$


The tensor $M_{\alpha \lambda}$ has 6 independent components, while $N_{\alpha \lambda}$ has only 3 . Actually, they completely characterize the traceless Ricci tensor:

$$
\tilde{R}_{\alpha \beta}=-2 M_{\alpha \beta}-4 * N_{\sigma(\alpha} u^{\sigma} u_{\beta}+M_{\sigma}^{\sigma}\left(g_{\alpha \beta}+2 u_{\alpha} u_{\beta}\right) .
$$

From the previous definitions, it is clear that

$$
N_{\alpha \beta}=R_{\alpha \beta} u^{\alpha} u^{\beta}=R=0 \Longrightarrow R_{\alpha \beta}=-2 M_{\alpha \beta},
$$

and this implies, in particular, that $R_{\alpha \beta} u^{\beta}=0$.

Let us finally give some formulae which is useful for the derivations of some expressions in Sect. 4:

$$
\begin{aligned}
T^{\alpha \beta \lambda \mu} u_{\alpha} u_{\beta} u_{\lambda} u_{\mu} & =E_{\alpha \beta} E^{\alpha \beta}+H^{\alpha \beta} H_{\alpha \beta}+M_{\alpha \beta} M^{\alpha \beta}+N_{\alpha \beta} N^{\alpha \beta}+\frac{R^{2}}{48}, \\
\left(\tilde{R}_{\alpha \rho} u^{\rho}\right)\left(\tilde{R}_{\sigma}^{\alpha} u^{\sigma}\right) & =2 N_{\sigma \rho} N^{\sigma \rho}-\left(M_{\sigma}^{\sigma}\right)^{2} \\
\tilde{R}_{\sigma \rho} \tilde{R}^{\sigma \rho} & =4 M_{\sigma \rho} M^{\sigma \rho}-4 N_{\sigma \rho} N^{\sigma \rho} .
\end{aligned}
$$

\section{B Electromagnetic case}

For space-times with an electromagnetic energy-momentum content, Penrose and Rindler [6] gave the following modification of the Bel-Robinson tensor (see [6] for the spinor notations and conventions):

$$
\begin{aligned}
t_{\alpha \beta \lambda \mu}= & \Psi_{A B C D} \bar{\Psi}_{A^{\prime} B^{\prime} C^{\prime} D^{\prime}}- \\
& -2 \gamma \nabla_{C D^{\prime}} \varphi_{A B} \nabla_{C^{\prime} D} \bar{\varphi}_{A^{\prime} B^{\prime}}+6 \gamma \nabla_{D\left(A^{\prime}\right.} \varphi_{(A B} \nabla_{C)\left|D^{\prime}\right|} \bar{\varphi}_{\left.B^{\prime} C^{\prime}\right)},
\end{aligned}
$$

where $\Psi_{A B C D}$ and $\varphi_{A B}$ are the Weyl and the electromagnetic spinors respectively, and $\gamma$ is the gravitational constant. This tensor is symmetric and traceless in the first three indices and has zero covariant derivative with respect to the last one, provided that the Einstein field equations hold:

$$
\begin{aligned}
\nabla^{\mu} t_{\alpha \beta \lambda \mu} & =0, \\
t_{\alpha \beta \lambda \mu}=t_{(\alpha \beta \lambda) \mu}, \quad t_{\alpha \lambda \mu}^{\alpha} & =0 .
\end{aligned}
$$

It is important to notice that the second part of this tensor is formed with $(\nabla F)^{2}$ terms, so it cannot be expressed by means of the Ricci tensor and its derivatives. Therefore it is independent from the tensors considered above.

The tensorial expression for (18) is rather involved but, in this case, it is useful in order to proof a positivity property. It should be noted here that in the final result we have returned to the initial signature metric $(-,+,+,+)$, and units such that $8 \pi \gamma=c=1$. With these conventions:

$$
\begin{aligned}
t_{\alpha \beta \lambda \mu}= & \frac{1}{4} \mathcal{T}_{\alpha \beta \lambda \mu}+2 \nabla_{(\alpha} F_{|\sigma| \beta} \nabla_{\lambda)} F_{\mu}^{\sigma}+2 \nabla_{(\alpha} F_{|\sigma| \beta} \nabla^{\sigma} F_{\lambda) \mu}- \\
& -\frac{1}{2} \nabla_{(\alpha} F_{|\sigma \rho|} \nabla_{\beta} F^{\sigma \rho} g_{\lambda) \mu}+\nabla_{\sigma} F_{\rho(\alpha} \nabla^{\rho} F_{\beta}^{\sigma} g_{\lambda) \mu}-g_{(\alpha \beta} \nabla^{\sigma} F_{\lambda)}^{\rho} \nabla_{\rho} F_{\sigma \mu},
\end{aligned}
$$


where $\mathcal{T}_{\alpha \beta \lambda \mu}$ is the Bel-Robinson tensor and $F_{\alpha \beta}$ is the electromagnetic tensor. From this expression it is easily seen that (19) satisfies the following positivity property:

$$
\begin{aligned}
& t_{\alpha \beta \lambda \mu} u^{\alpha} u^{\beta} u^{\lambda} u^{\mu} \geq 0, \forall \vec{u}, u_{\mu} u^{\mu}<0 \\
& t_{\alpha \beta \lambda \mu} u^{\alpha} u^{\beta} u^{\lambda} u^{\mu}=0 \Longleftrightarrow\left\{\begin{array}{l}
\mathcal{T}_{\alpha \beta \lambda \mu} u^{\alpha} u^{\beta} u^{\lambda} u^{\mu}=0 \Longleftrightarrow C_{\alpha \beta \lambda \mu}=0, \\
\dot{F}_{\lambda \mu} \equiv u^{\alpha} \nabla_{\alpha} F_{\lambda \mu}=0
\end{array}\right.
\end{aligned}
$$

To prove this, let us introduce the orthogonal projector to $\vec{u}, h_{\alpha \beta} \equiv g_{\alpha \beta}+u_{\alpha} u_{\beta}$. Then, we compute $t_{\alpha \beta \lambda \mu} u^{\alpha} u^{\beta} u^{\lambda} u^{\mu}$ :

$$
\begin{aligned}
t_{\alpha \beta \lambda \mu} u^{\alpha} u^{\beta} u^{\lambda} u^{\mu} & =\frac{1}{4} \mathcal{T}_{\alpha \beta \lambda \mu} u^{\alpha} u^{\beta} u^{\lambda} u^{\mu}+2 u^{\alpha} u^{\beta} \dot{F}_{\sigma \alpha} \dot{F}_{\beta}^{\sigma}+\frac{1}{2}\left(\dot{F}_{\sigma \rho} \dot{F}^{\sigma \rho}\right)= \\
& =\frac{1}{4} \mathcal{T}_{\alpha \beta \lambda \mu} u^{\alpha} u^{\beta} u^{\lambda} u^{\mu}+\left(h^{\alpha \sigma} \dot{F}_{\alpha \beta} u^{\beta}\right)\left(h_{\lambda \sigma} \dot{F}^{\lambda \mu} u_{\mu}\right)+ \\
& +\frac{1}{2}\left(h^{\alpha \sigma} h^{\beta \rho} \dot{F}_{\alpha \beta}\right)\left(h_{\lambda \sigma} h_{\mu \rho} \dot{F}^{\lambda \mu}\right) \geq 0
\end{aligned}
$$

and the equality holds only when $C_{\alpha \beta \lambda \mu}=0$ and

$$
h^{\alpha \lambda} h^{\beta \mu} \dot{F}_{\lambda \mu}=0, \quad h^{\alpha \sigma} \dot{F}_{\alpha \beta} u^{\beta}=0 \Longleftrightarrow \dot{F}_{\alpha \beta}=0 .
$$

\section{References}

[1] L. Bel, Sur la radiation gravitationnelle, C.R. Acad. Sci. Paris 247, 1094-1096 (1958).

[2] B. Mashhoon, J.C. McClune and H. Quevedo, Gravitational superenergy tensor, Phys. Lett. A 231, 47-51 (1997).

[3] L. Bel, Introduction d'un tenseur du quatrième ordre, C.R. Acad. Sci. Paris 248, 1297-1300 (1959).

[4] R.K. Sachs, Propagation laws for null and type III gravitational waves, Z. Physik 157, 462-477 (1960).

[5] C.D. Collinson, A class of conserved tensors in an arbitrary gravitational field, Proc. Cambridge Phil. Soc. 58, 346-362 (1962).

[6] R. Penrose and W. Rindler, Spinors and space-time, Cambridge University Press, Cambridge (1984).

[7] M.Á.G. Bonilla and J.M.M. Senovilla, Some Properties of the Bel and BelRobinson Tensors, Gen. Rel. Grav. 29, 91-116 (1997).

[8] D. Kramer, H. Stephani, E. Herlt and M.A.H. MacCallum, Exact Solutions of Einstein's Field Equations, Cambridge University Press, Cambridge (1980). 